\title{
Fundamentos Nightingaleanos, cuidado humano e políticas de saúde no Século XXI
}

\author{
Nightingale fundamentals, human care and health policies in the $21^{\text {st }}$ century \\ Fundamentos Nightingaleanos, atención humana y políticas de salud en el siglo XXI
}

\author{
Márcia de Assunção Ferreiral @ ; Priscilla Silva Machado" @ ; Marta Sauthierl @ ; Rafael Celestino da Silval @
}

'Universidade Federal do Rio de Janeiro, Rio de Janeiro, RJ, Brasil; "Universidade Federal do Espírito Santo, Votória, ES, Brasil

\begin{abstract}
RESUMO
Objetivo: estabelecer relações entre os fundamentos Nightingaleanos na sustentabilidade de uma clínica de cuidado humano em diálogo com conceitos que sustentam duas atuais políticas de saúde brasileiras. Conteúdo: a ciência da enfermagem se afirma em uma abordagem holística do ser humano, com vistas à saúde integral, ressaltando a pessoa na sua dimensão física, mental, emocional e espiritual. Este princípio sustenta práticas humanizadas de cuidado e também integrativas, na proposição de cuidados integrais centrados na pessoa e relacionamento terapêutico para promover a saúde e o bem-estar. As fontes principais foram a obra seminal de Nigthingale, teorias de enfermagem, textos aplicados da Política Nacional de Humanização e de Práticas Integrativas e Complementares de Saúde. Conclusão: os princípios básicos do cuidado propostos por Nightingale refletem-se em atuais políticas de saúde, contribuindo para ampliar a autonomia dos profissionais de enfermagem, na oferta de cuidados baseados em conceitos próprios, em favor de uma Enfermagem Integrativa e Humana.

Descritores: Teoria de Enfermagem; Cuidados de Enfermagem; Humanização da Assistência; Política Pública; Terapias Complementares.
\end{abstract}

\begin{abstract}
Objective: to establish relations between Nightingale fundamentals on the sustainability of human clinical care in dialogue with concepts underpinning two of Brazil's current health policies. Content: nursing science is grounded on a holistic approach of the human being, with a view to comprehensive health, emphasizing subjects in their physical, mental, emotional and spiritual dimensions. This principle underpins integrative, humanized care practices in proposing comprehensive care centered on the human person and a therapeutic relationship designed to promote health and wellbeing. The main sources were Nightingale's seminal work, nursing theories, and applied texts from Brazil's National Policy of Humanization and Integrative, Complementary Healthcare Practices. Conclusion: The basic health care principles proposed by Nightingale, reflected in current health policies, contribute to expanding the autonomy of nursing personnel in the providing care based on concepts specific to nursing, in favor of Integrative, Human Nursing.
\end{abstract}

Descriptors: Nursing Theory; Nursing Care; Humanization of Assistance; Public Policy; Complementary Therapies.

\section{RESUMEN}

Objetivo: establecer relaciones entre los fundamentos de Nightingale sobre la sostenibilidad de la atención clínica humana en diálogo con los conceptos que sustentan dos de las políticas de salud actuales de Brasil. Contenido: la ciencia de la enfermería se fundamenta en un enfoque holístico del ser humano, con miras a la salud integral, enfatizando los sujetos en sus dimensiones física, mental, emocional y espiritual. Este principio sustenta las prácticas de atención integral y humanizada al proponer una atención integral centrada en la persona humana y una relación terapéutica orientada a promover la salud y el bienestar. Las fuentes principales fueron el trabajo fundamental de Nightingale, las teorías de enfermería y los textos aplicados de la Política Nacional de Humanización y Prácticas de Atención Integrativa y Complementaria de Salud de Brasil. Conclusión: Los principios básicos del cuidado de la salud propuestos por Nightingale, reflejados en las políticas de salud vigentes, contribuyen a ampliar la autonomía del personal de enfermería en la prestación de cuidados basados en conceptos propios de la enfermería, a favor de la Enfermería Integrativa, Humana.

Descriptores: Teoría de Enfermería; Atención de Enfermería; Humanización de la Atención; Política Pública; Terapias Complementarias.

\section{INTRODUÇÃO}

Em sua obra seminal, Florence Nightingale define a enfermagem como arte e expressa que para ser arte, a Enfermagem requer uma devoção exclusiva e um rigoroso preparo. Nesta obra Nightingale se dirige às mulheres que, naquela época, eram as que se responsabilizavam pelo cuidado, pela saúde de alguém, ressaltando que o conhecimento sanitário era primordial para salvaguardar as pessoas do adoecimento ou para recuperá-las ${ }^{1}$.

Observa-se que nesta obra, Nightingale assevera que a arte da enfermagem deveria incluir condições tais que, por si, possibilitassem o que ela entendia ser a assistênica de enfermagem. Além do que, Nightingale faz uma crítica quando escreve que a arte da enfermagem, como era praticada, não contribuía para que a doença exercesse o papel de ser um processo restaurador, como pensada por Deus ${ }^{1}$. Nos seus escritos não há propriamente um conceito de arte, mas a

Autor correspondente: Márcia de Assunção Ferreira. E-mail: marcia.eean@gmail.com.

Editor responsável: Sonia Acioli de Oliveira. 
arte da enfermagem reiteradamente aludida por ela se expressa em o que e como fazer para atender as pessoas a manter ou recuperar a saúde, ou seja, a arte da enfermagem se expressa nas práticas de cuidar e nos cuidados realizados.

A partir de sua obra inaugura-se o saber profissional da enfermagem e os princípios do cuidado sustentado em uma teoria ambientalista que lhe dá consistência para a construção de sua ciência. As condições e arranjos necessários a serem realizados no o que (cuidados) e como fazer (cuidar) se pauta em deixar a pessoa nas melhores condições para que a natureza possa agir e nela se instaurar o processo restaurador, sendo, portanto, primordial para um cuidado pleno e singular. Deriva-se daí um conjunto de elementos essenciais à enfermeira e ao cuidado, que Nightingale disserta com foco na pessoa a ser cuidada ${ }^{1}$

O cuidado preconizado por Nightingale requer capacidade intelectual da enfermeira para observar com profundidade e descrever com propriedade aquilo que observou, mas também é preciso que a enfermeira se antecipe às necessidades do paciente, o que requer que o compreenda profundamente ${ }^{1}$. Tais princípios norteadores centram o cuidado na pessoa e no ambiente que a cerca, exigindo o manejo de seu entorno e contorno, valorizando elementos naturais, os objetos, a estética e as pessoas que dão dinamicidade ao ambiente, incluindo as maneiras de cuidar dos pacientes e as visitas que lhes são feitas - ou seja, valoriza os atos humanos. Valoriza-se o equilíbrio no cuidado, evidenciando que tanto as faltas quanto os excessos são dispensáveis se a finalidade for promover saúde, conforto e bem-estar.

$\mathrm{Na}$ análise da teoria ambientalista e dos princípios básicos que norteiam a ação da enfermeira no cuidado de enfermagem, evidenciam-se estímulos naturais de cura, escuta atenta e acolhedora, formação de vínculo e integração da pessoa a ser cuidada ao meio que a cerca, considerando as suas influências no processo de cuidado/cura/saúde. Além de considerar as influências do corpo na mente humana ${ }^{1}$.

No atual contexto histórico do século XXI, o cuidado de Enfermagem ocorre no cotidiano de uma prática profissional, com intenção terapêutica, resulta de processos de trabalho que objetivam aliviar, confortar, promover o bem-estar, a saúde, prevenir agravos, tratar e/ou reabilitar a pessoa para (re)integrá-la ao seu cotidiano ${ }^{2-3}$. Observa-se, à luz dos seus princípios, que na perspectiva Nightingaleana o cuidado é de natureza integradora e ecológica. Portanto, os princípios fundamentais da enfermagem, cunhados no século XIX permanecem fundamentando a atuação efetiva sobre o ambiente e as relações que se estabelecem a partir dele.

Reconhece-se que a prática da enfermagem, cientificamente fundamentada, requer conhecimentos assentados nas ciências, nas técnicas e tecnologias necessárias para responder às situações de saúde e doença; contudo, as pessoas também expectam por atenção, cuidados compassivos e comunicação efetiva, que são qualidades referidas como expressões da arte da enfermagem ${ }^{3}$, de seu o que e como fazer. Corresponder a estas expectativas das pessoas é valorizado e personaliza o cuidado no atendimento das suas necessidades biopsicossociais, espirituais e preferências culturais $^{4}$, vindo ao encontro da compreensão do ser humano em componentes físicos, intelectuais, emocionais, sociais e espirituais, à luz dos escritos Nightingaleanos.

Em um debate sobre as dimensões da ciência e da arte da enfermagem, reconhece-se o lugar do conhecimento científico na orientação das diretrizes clínicas, da competência técnica, das práticas baseadas em evidências geradas pela ciência, mas as autoras observam que a expressão da sensibilidade, a leveza, o carinho e a gentileza nos comportamentos e toques, a expressão de emoções geram ações intangíveis que afetam os cuidados e expressam a sua dimensão arte $^{3-5}$, vindo ao encontro do que ressaltam as Notas sobre Enfermagem de Nightingale, quando alude às maneiras de cuidar da enfermeira.

Este artigo se caracteriza como um ensaio teórico, e objetiva estabelecer relações entre os fundamentos Nightingaleanos, expressos na ciência e na arte da enfermagem, na sustentabilidade de uma clínica de cuidado humano, em diálogo com conceitos que sustentam duas políticas de saúde brasileiras do século XXI.

\section{CONTEÚDO}

\section{Fundamentos Nightingaleanos: bases para a ciência e aplicação na clínica do cuidado humano}

Na segunda metade do século XIX e no século XX, a enfermagem se construiu como ciência, e neste século XXI, a ciência da enfermagem vem se expressando como uma ciência de saberes plurais ${ }^{6}$ e se reafirmando com uma abordagem holística do ser humano. As teorias de enfermagem até então produzidas assumem o humano como ser integral, expresso em corpo, mente e espírito ${ }^{7}$, situado em um contexto sócio histórico e cultural. O desenvolvimento de uma visão holística do ser humano esteia-se na cosmovisão unitária da disciplina de Enfermagem, cuja filosofia orienta a concepção de unidade corpo-mente-espírito para promoção da saúde integral e do cuidado humano, para alcançar a paz, o bem-estar e a cura ${ }^{8-9}$. 
A saúde integral viabiliza a aplicação do potencial humano na sua condição biológica, psíquica e social, numa perspectiva de equilíbrio vital. Assim, a pessoa contribui para o desenvolvimento social, sendo a saúde, portanto, uma questão de direito, normativo e bioético ${ }^{10}$. Nesse sentido, cuidar dos indivíduos implica cuidar da sociedade e vice-versa. Por isso importa que a pessoa seja vista de forma holística e não fragmentada.

Cuidar na enfermagem exige saberes e fazeres fundamentados em conhecimentos, e os desenvolvimentos conceituais e teóricos representam ou correspondem às mudanças paradigmáticas, aos avanços nas reflexões éticas, bioéticas e epistemológicas ocorridas na área, interferindo e atualizando-se nas concepções do metaparadigma saúde, ambiente, pessoa e enfermagem, que compõem as teorias de Enfermagem.

Tais avanços são importantes para a compreensão do cuidado, cujo termo é polissêmico e precisa ser contextualizado nos campos da moral, da ética, da filosofia e da socioantropologia. Abrange significados de ações voltadas para si, para o outro e o coletivo, e de manutenção de coisas/objetos e tecnologias próprias aos ambientes de cuidado $^{11-12}$. Cuidado que também ganha significado na solidariedade, nas ações intersociais, na obrigação moral, religiosa e/ou espiritual de ir ao encontro do outro para ajudá-lo quando necessário ${ }^{13}$.

$\mathrm{O}$ ato de cuidar ocorre na relação entre pessoas, construída com empatia, por meio da sensibilidade e capacidade de comunicação, para que haja percepção das necessidades do outro, nos seus contextos próprios de valores sociais, humanos, filosóficos e espirituais ${ }^{14}$. Na essência, o cuidado é ético e o saber cuidar se traduz pela ética humana, considerada como o conjunto de valores que envolvem a solidariedade, o amor, o desvelo em uma visão da integralidade, espiritualidade e ecologia. Expressa a humanidade, os valores e princípios morais das pessoas, o comportamento do ser humano para com os outros ${ }^{15}$.

Os paradigmas biomédico e emergente se evidenciam em várias teorias de enfermagem. Teorias do modelo humanista, integracionista e da complexidade relevam os processos da natureza, da sociedade e da história nas análises sobre o processo saúde-doença-cuidado. Na perspectiva da produção social da saúde o contexto sociocultural ganha expressão, bem como a integralidade biopsicossocial, relevando a subjetividade nas diversas expressões do humano ${ }^{16}$, direcionando as pesquisas que envolvem o cuidado na sua epistemologia e práxis.

Os atos de cuidado na enfermagem exigem fundamentação teórica, efetivam-se nas técnicas procedimentais, em uma complexa relação humana que implica em intersubjetividade, interação sensível e empática entre seus partícipes, distanciando-se do paradigma biomédico focado na doença e aproximando-se do da saúde. Diferenciam-se dos cuidados de outros profissionais, abrangem a solicitude, a compaixão, a disponibilidade, com intencionalidade organizada e integrada ${ }^{17}$. A compreensão do valor do cuidado enquanto ato do profissional de Enfermagem depende das concepções éticas do sentido da vida, que levam o Enfermeiro, como indivíduo e profissional, a valorizar e respeitar a própria existência e a do outro. O cuidado possibilita ao enfermeiro realizar-se, exercer sua unicidade, seu modo de existir, uma vez que o cuidado é por princípio e natureza ético. O exercício de suas habilidades e competências oportuniza ao enfermeiro compreender ontologicamente a unicidade do outro e ajudá-lo a viver à luz dos sentidos que ele atribui à sua vida/existência9,18.

Cuidar das pessoas nas suas expressões biológicas, psicológicas, mentais, sociais e espirituais implica em aplicar os princípios holistas nos processos de saúde e adoecimento; retomar a visão de uma cosmologia integradora da natureza e do ser humano em defesa do equilíbrio, considerando o meio ambiente, natural e social, no estabelecimento de diagnósticos ${ }^{19}$, em voga nos discursos e políticas de saúde atuais.

Na concepção da plenitude do ser, a espiritualidade se avoluma e se torna campo de ação, haja vista as produções científicas que evidenciam a atuação da religiosidade/espiritualidade na saúde mental, enfrentamentos e adaptações aos problemas de saúde ${ }^{20}$. Na atenção às necessidades espirituais para integrá-las no atendimento aos pacientes, recomenda-se seguir por caminhos sensíveis. Compreende-se que o atendimento desta dimensão prescinda de tecnologias duras, requerendo a presença genuína do ser humano, sua sensibilidade e motivação.

Evidenciam-se teóricas que discutem a espiritualidade como tema central (Newman, Neuman, Parse, Watson) ${ }^{21}$, ou periférico (Levine, Roy, Leininger, Rogers e Horta) em seus fundamentos. Nightingale também releva a espiritualidade no âmbito da natureza humana, sendo um recurso potente ${ }^{21}$, revelando um dos princípios básicos Nightingaleanos: compreender profundamente o ser humano ${ }^{1}$.

Consoante ao preceito de Nightingale de que os cuidados de enfermagem devem auxiliar o paciente a reaver o equilíbrio para promover a sua cura, a enfermagem objetiva ajudar as pessoas a terem harmonia na mente, no corpo e na alma, pois, assim, os processos de autoconhecimento, autorrespeito, autocura e autocuidado ocorrem viabilizando a diversidade ${ }^{22}$.

Enxergar o outro e suas manifestações, e também suas respostas humanas, na linguagem atual diagnóstica, envolve saber o quê procurar. Nesse sentido, retoma-se a afirmação de Nightingale de que à enfermeira cabe observar 
com profundidade e descrever com propriedade. Aprofundar o olhar implica em atentar para o que deve ser visto, como deve ser identificado e seus significados para a clínica da enfermagem.

Na clínica do cuidado de enfermagem têm-se particularidades que implicam considerar o metaparadigma que sustenta conceitualmente a disciplina no campo da ciência: ser humano, ambiente, saúde e a própria enfermagem como campo de saberes e de práticas. No âmbito dessa clínica, a presença dos envolvidos no cuidado, a comunicação efetiva no plano de uma relação dialógica que fundamente a interação e a vivência da subjetividade implicada nas relações interpessoais são elementos que importam e conformam seu marco conceitual ${ }^{23}$ e reafirmam o que se assume como a dimensão da arte no cuidado de enfermagem: a técnica, a intuição e a sensibilidade ${ }^{3,24}$, cujos saberes postos em ação são únicos e diferenciados de enfermeiro para enfermeiro ${ }^{24}$.

Enfermagem é disciplina prática, se faz na ação cotidiana da atenção e da intenção a outrem, é campo de uma ciência e de uma arte de cuidar, portanto, trabalhar com os recursos atuais das técnicas e das tecnologias, amparados em seus fundamentos primeiros e nos conhecimentos gerados com os avanços da ciência, atende aos propósitos de uma enfermagem humana, ética e solidária, uma enfermagem profissional que busca nos estudos e nas pesquisas as melhores evidências para cuidar, com segurança, eficiência e eficácia na promoção da saúde humana.

\section{Princípios Nightingaleanos: interfaces com duas políticas de saúde atuais}

Ao se refletir sobre os conceitos de cuidado e situá-los no campo da saúde, considerando os princípios básicos do cuidado humano que abrange a observação, a descrição e a compreensão do ser humano, resgatam-se os fundamentos da enfermagem em seus princípios genuínos, dando-Ihe lugar de destaque nas atuais políticas de saúde, o que contribui para ampliar as possibilidades de autonomia nas ações dos profissionais de enfermagem.

Como exemplo, observa-se que o saber difundido por meio da teoria ambientalista de Nightingale se expressa claramente na Política Nacional de Humanização (PNH), uma política de governo brasileira criada em 2003, em que o conceito de ambiente sustenta práticas de cuidado, na salvaguarda de uma ambiência que ofereça condições para que as famílias estejam presentes junto aos seus familiares hospitalizados, de modo a viabilizar o vínculo e o protagonismo dos usuários, no intento de melhores resultados nos tratamentos que lhe são ofertados ${ }^{25}$.

A defesa do cuidado no âmbito da ação da enfermeira articulada aos mecanismos naturais de cura e de recuperação da saúde, mobilizando as forças vitais do ser humano, presentes no discurso Nightngaleano ${ }^{1}$, também se evidenciam em outra política brasileira lançada em 2006, a Política Nacional de Práticas Integrativas e Complementares em Saúde. Tal política vem ampliando seu campo de implementação, especialmente na atenção primária de saúde, com uma variedade de práticas de promoção da saúde pautadas na interdisciplinaridade ${ }^{26}$.

No âmbito desta política, as Práticas Integrativas e Complementares em Saúde (PICS) enfatizam a necessária atenção centrada no ser humano, com estímulo à participação dos usuários no cuidado dando-lhes mais protagonismo, promovendo melhor relacionamento terapêutico, valorização das singularidades, saberes e potencial de autocura ${ }^{26}$, requerendo dos profissionais algumas qualidades para que tais premissas sejam atendidas.

Na defesa de uma clínica de cuidado, aplicam-se as bases fundamentais de sustentação das práticas de cuidar distintivas da enfermeira, em que os conhecimentos gerais se apliquem, mas a linguagem disciplinar específica permaneça para que a orientação disciplinar da enfermagem objetive e dê visibilidade aos atos profissionais da enfermagem, não desvirtuando-a de seu rumo ${ }^{9}$, traçado desde 1859.

Na intenção de um atendimento humano, requerido pela PNH e pelas PICS, observar com profundidade, descrever com propriedade e compreender profundamente o ser humano são competências a serem desenvolvidas pela enfermeira ${ }^{1}$, o que exigirá atenção e presença plena no cuidado. Criar vínculo e estabelecer uma relação terapêutica são princípios do cuidado humano, na perspectiva Nightingaleana.

Os fundamentos apresentados vêm ao encontro das bases dos cuidados do que neste século se convencionou chamar de Enfermagem Integrativa ${ }^{27}$, que vem mobilizando produções e práticas de cuidados no Brasil e em diversos países, cuja estrutura conceitual e prática se voltam à prestação de cuidados centrados na pessoa, baseados em construção de boas relações para que se alcance a saúde das populações, se obtenha qualidade e satisfação do usuário no atendimento e também do trabalhador, com redução de custos ${ }^{27}$, além de corroborar a integralidade da assistência em saúde.

A integralidade da assistência, um dos princípios do Sistema Único de Saúde, se viabiliza por meio da atuação multidisciplinar, se expressa na incorporação de atitudes de respeito e cidadania pelos sujeitos em relação. Em favor desta integralidade, defende-se que a Enfermagem incorpore práticas transformadoras, convergentes com a Enfermagem Integrativa, que traz em sua aplicação avanços epistemológicos, históricos, éticos, estéticos, empíricos, pessoais e políticos, desde seus primórdios ${ }^{27}$. 
A Enfermagem Integrativa desenvolve-se epistemologicamente, inclusive resgatando da sua formação Nigthingaleana o que é macro na área. Converge com a PNH, que requer a compreensão da subjetividade humana, ampliando o olhar da ciência e resgatando os profissionais, especialmente de enfermagem, do contexto micro na área.

Um princípio fundamental do pensamento Nightingaleano é o cuidado dos seres humanos e não das doenças, para que tenham condições de viver e serem saudáveis. Para que este princípio se faça, cuida-se do entorno e do contorno - do ambiente onde as pessoas estão, atentando-se para a variedade, tão necessária ao resguardo da saúde mental, pois a monotonia é fator desencadeante de stress; do silêncio e do ruído que pode trazer desconforto físico e mental ${ }^{1}$. Cuida-se para que não haja sobrecarga psíquica, que desajusta os humores e desencadeia reações prejudiciais ao equilíbrio orgânico, nisto se inclui a influência dos pensamentos, sentimentos e das emoções no processo de recuperação do paciente e na manutenção de sua saúde, da presença danosa do outro, tratada como visitas prejudiciais ao processo de recuperação do paciente ${ }^{1}$.

O conceito de cuidado é polissêmico e abrange múltiplas dimensões compreendidas no campo da técnica, da clínica, dos encontros e das relações entre humanos e suas atitudes, das relações institucionais e políticas ${ }^{28}$. Ao circunscrevê-lo no âmbito de um fazer profissional, como no da enfermagem, tais dimensões estarão presentes e amparadas nos padrões de conhecimento da enfermagem ${ }^{6}$.

O cabedal do conhecimento Nightingaleano se atualiza nos discursos atuais, evidenciando sua posição visionária no que se consideram como "novidades" do século XXI, nas políticas de saúde em que os conceitos de ambiente e de ambiência se destacam na promoção do acolhimento e na eficácia dos tratamentos - os espaços do cuidado (tanto físico quanto social tornam-se importantes aliados da saúde); a mente e a espiritualidade precisam ser cuidadas tanto quanto o corpo; a biologia se entrelaça com a psique, a matéria com o espírito e as boas relações pessoais são fatores protetores e promotores de saúde. Reconhecemos, portanto, os conteúdos nucleares da disciplina Enfermagem, no que pese seus fundamentos.

A vanguarda do pensamento Nightingaleano e sua atualização, articulado aos princípios, conceitos e políticas de saúde contemporâneas evidenciam suas contribuições e sua posição visionária frente à saúde e ao cuidado.

\section{CONCLUSÃO}

Retomar o discurso Nightingaleano refletindo-se sobre os fundamentos do cuidado em favor de uma Enfermagem do século XXI é valorar o que de genuíno se tem no conhecimento da enfermagem, amparado no seu metaparadigma e na sua proposição primeira na qual se pensou o que é e o que não é enfermagem, inaugurando um campo pleno de possibilidades para atuação em favor do humano e da humanidade.

Estas possibilidades também se promovem pela definição da enfermagem como arte e ciência do cuidado. A arte expressa no o que e como fazer da enfermagem nos dá oportunidades para transcender na prática, pois agrega habilidade, conhecimento, ação, consciência, percepção, ideias, ideais, razão, emoção na produção de técnicas, tecnologias e de expressões. A arte é uma atividade humana que envolve presença, equilíbrio, harmonia, estética, sensibilidade - reflete o humano na sua condição inteira em um fazer que não se repete, nesse sentido, a enfermagem como arte tem um fazer único e diferenciado que se constrói e se (re)constrói no momento da presença com o usuário. A promissora ciência do século XX nos fez avançar como disciplina, e a arte vem nos dando a possibilidade de criar e transcender e com isso, proporcionar à enfermagem oportunidades para crescer ainda mais neste século XXI.

\section{REFERÊNCIAS}

1. Nightingale F. Notas sobre Enfermagem o que é e o que não é. São Paulo: Cortez; 1989.

2. Carnut L. Care, integrality and primary care: essential articulation to reflect on the health sector in Brazil. Saúde em Debate. [Internet]. 2017 [cited 2019 Dec 10]; 41(115):1177-86. DOI: https://doi.org/10.1590/0103-1104201711515

3. Duarte NE, Ferreira MA, Lisboa MTL. Social representations of nursing care and their relationship with learning strategies. Intervozes: trabalho, saúde, cultura. [Internet]. 2019 [cited 2020 Mar 29]; 4(2):24-35. Available from: http://www.fmpfase.edu.br/intervozes

4. Palos GR. Care, compassion, and communication in Professional Nursing: art, science, or both. Clin. J. Oncol. Nurs. [Internet] 2014 [cited 2020 Jun 01]; 18(2):247-8. DOI: https://doi.org/10.1188/14.CJON.247-248

5. Vega H, Hayes K. Blending the art and science of Nursing. Nursing. [Internet]. 2019 [cited 2020 Jun 01]; 49(9):62-3. DOI: https://doi.org/10.1097/01.NURSE.0000577752.54139.4e

6. Jasmine T. Art, Science, or Both? Keeping the Care in Nursing. Nurs. Clin. North Am. [Internet]. 2009 [cited 2020 Jun 01 ] 44(4):415-21. DOI: https://doi.org/10.1016/j.cnur.2009.07.003

7. Queirós PJP. Enfermagem, uma ecologia de saberes. Cul cuid. [Internet]. 2016 [cited 2020 Jun 01]; 20(45):137-56. DOI: https://doi.org/10.14198/cuid.2016.45.15 
8. Pinto AC, Garanhani ML, França TE, Pierotti I. The concept of the human being in nursing theories: establishing a relationship with the teaching of the human condition. Pro-Posições. [Internet]. 2017 [cited 2019 Dec 10]; 28(Suppl. 1):88-110. DOI: https://doi.org/10.1590/1980-6248-2015-0164

9. McMillan E, Stanga N, Van Sell SL. Holism: a concept analysis. Int. J. Nurs. Clin. Pract. [Internet]. 2018 [cited 2019 Dec 10]; 5:582. DOI: https://doi.org/10.15344/2394-4978/2018/282

10. Watson J. Clarifying the discipline of nursing as foundational to development of professional nursing. Texto contexto - enferm. [Internet]. 2017 [cited 2019 Dec 17]; 26(4):editorial. DOI: http://dx.doi.org/10.1590/0104-07072017002017editorial4

11. D'Andrea G, Rodriguéz AMMM, Ventura CAA, Mishima SM. Right to health: A concept proposal for the operationalization of qualitative research. R. Dir. sanit. [Internet]. 2017 [cited 2020 Jan 15]; 18(1):57-74. DOI: http://dx.doi.org/10.11606/issn.23169044.v18i1p57-74

12. Baggio MA, Erdmann AL. The circularity of the processes of caring and being cared for in shaping the care for "the we". Rev. Enf. Ref. [Internet]. 2015 Dez [cited 2020 Jan 6]; serIV(7):11-20. DOI: http://dx.doi.org/10.12707/RIV15012

13. Monteiro APTAV, Curado Manuel. A new epistemology of nursing: a post-human care?. Rev. Enf. Ref. [Internet]. 2016 [cited 2020 Jan 6]; serIV(8):141-8. DOI: http://dx.doi.org/10.12707/RIV15069

14. Mascarenhas YS, Cunha CN, Fernandes CL, Santos RM, Morais IF. Care and their dimensions: a bibliographic review. TF [Internet]. 2017 [cited 2020 Feb 15]; 10(1):85-106. Available from: http://natal.uern.br/periodicos/index.php/RTF/article/view/tf.v10i1.3064

15. Savieto RM, Leão ER. Nursing assistance and Jean Watson: a reflection on empathy. Esc. Anna Nery [Internet]. 2016 [cited 2020 Jan 06]; 20(1):198-202. Available from: https://www.scielo.br/scielo.php?pid=S1414$81452016000100198 \&$ script=sci_arttext\&ting=en

16. Gonçalves, AS. The eco-pedagogy care in Leonardo Boff. Revista Caminhando [Internet]. 2016 [cited 2020 Feb 20]; 21(1):11523. DOI: http://dx.doi.org/10.15603/2176-3828/caminhando.v21n1p115-123

17. Anderson MIP, Rodrigues RD. O paradigma da complexidade e os conceitos da Medicina Integral: saúde, adoecimento e integralidade. Revista Hospital Universitário Pedro Ernesto [Internet] 2016 [cited 2020 Feb 20]; 15(3):242-52. DOI: https://doi.org/10.12957/rhupe.2016.29450

18. Queirós PJP. Caring: from condition of human existence to professionalised integral care. Rev. Enf. Ref. [Internet]. 2015 [cited 2020 Abr 14]; serIV(5):139-46. DOI: http://dx.doi.org/10.12707/RIV14079

19. Silveira DR, Gradim FJ. Viktor Frankl's contributions for the colective health movement. Revista da Abordagem Gestáltica [Internet]. 2015 [cited 2020 Feb 20]; 21(2):153-61. Available from: http://pepsic.bvsalud.org/scielo.php?script=sci_arttext\&pid=S1809-68672015000200005\&lng=pt\&tlng=pt

20. Telesi Júnior E. Práticas integrativas e complementares em saúde, uma nova eficácia para o SUS. Est. av. [Internet]. 2016 [cited 2020 Jan 5]; 30(86):99-112. DOI: https://dx.doi.org/10.1590/S0103-40142016.00100007

21. Koenig HG. Religion, spirituality, and health: a review and update. Adv Mind Body Med. [Internet]. 2015 [cited 2020 Jan 5]; 29(3):19-26. Available from: https://www.ncbi.nlm.nih.gov/pubmed/26026153

22. Figueiredo NMA. Espiritualidade no espaço do cuidado: questões objetivas no plano da subjetividade. Enfermería Universitaria [Internet]. 2016 [cited 2020 Jan 18]; 13(1):1-2. DOI: https://dx.doi.org/10.1016/J.reu.2016.01.004

23. Watson J. Florence Nightingale and the enduring legacy of transpersonal human caring-healing. J. Holist. Nurs. [Internet]. 2010 [cited 2020 Feb 20]; 28(1):107-8. DOI: http://dx.doi.org/10.1177/0898010110361779

24. Silva RCS, Ferreira MA, Apostolidis T, Brandão MAG. A conceptual framework of clinical nursing care in intensive care. Rev. Latino-Am. Enfermagem [Internet]. 2015 [cited 2019 Dec 15]; 23(5):837-45. DOI: http://dx.doi.org/10.1590/01041169.0501 .2622

25. Queirós PJP. Reflections for a nursing epistemology. Texto Contexto Enferm. [Internet]. 2014 [cited 2020 Jul 02 ]; $23(3): 776-81$. DOI: http://dx.doi.org/10.1590/0104-07072014002930013

26. Freitas FDS, Silva RN, Araújo FP, Ferreira MA. Environment and humanization: resumption of nightingale's discourse in the national humanization policy. Esc. Anna Nery [Internet]. 2013 [cited 2019 Dec 15]; 17(4):654-60. Available from: https://www.scielo.br/scielo.php?script=sci_arttext\&pid=S1414-81452013000400654

27. Tesser CD, Sousa IMC, Nascimento MC. Traditional and Complementary Medicine in Primary Health Care in Brazil. Saúde em Debate [Internet]. 2018 [cited 2019 Dec 15]; 42(spe1):175-88. DOI: https://doi.org/10.1590/0103-11042018S112

28. Kreitzer MJ. Integrative nursing: application of principles across clinical settings. Rambam Maimonides Med J. [Internet] 2015 [cited 2020 Jan 5]; 6(2):e0016. DOI: http://dx.doi.org/10.5041/RMMJ.10200

29. Alves CA. Healthcare in Brazil: spaces of creation, struggles, and challenges. Cad. Saúde Pública [Internet]. 2019 [cited 2020 Jul 03]; 35(8):e00128519. DOI: https://doi.org/10.1590/0102-311x00128519 